Voix et Images

voixetimages

\title{
Bibliographie de France Théoret
}

\section{Hélène Girard}

Volume 14, numéro 1 (39), automne 1988

France Théoret : narratrice de la subjectivité

URI : https://id.erudit.org/iderudit/200753ar

DOI : https://doi.org/10.7202/200753ar

Aller au sommaire du numéro

Éditeur(s)

Université du Québec à Montréal

ISSN

0318-9201 (imprimé)

1705-933X (numérique)

Découvrir la revue

Citer ce document

Girard, H. (1988). Bibliographie de France Théoret. Voix et Images, 14(1), 57-69.

https://doi.org/10.7202/200753ar d'utilisation que vous pouvez consulter en ligne.

https://apropos.erudit.org/fr/usagers/politique-dutilisation/ 


\section{Bibliographie de France Théoret}

\section{par Hélène Girard, Université du Québec à Montréal}

\section{I - Cuvres de France Théoret}

\section{I.1 - Volumes}

Bloody Mary, Montréal, les Herbes rouges, 1977, 24 p. [extraits parus dans la Poésie québécoise des origines à nos jours, Montréal, PUQ/l'Hexagone, 1980, p. 521-522, et dans La bolsa y la vida ediciones, 1981 (traduction de Lazlo Moussong, Sara Diazmunoz, Brigitte Morissette)].

Une voix pour Odile, Montréal, les Herbes rouges, coll. «Lecture en vélocipède», 1978, 76 p. [extraits parus dans la Barre du jour, no 50, hiver 1975, p. 30-36 et dans Change, nos 30-31, 1977, p. 143-145].

Vertiges, Montréal, les Herbes rouges, 1979, 38 p.

Nécessairement putain, Montréal, les Herbes rouges, 1980, 52 p. [extraits parus dans Cuadernos de Literatura nueva epoca, 1983 p. $45-46$ et dans Plural, no 159, décembre 1984, p. 14 (traduction de Nicole Mercier)].

Nous parlerons comme on écrit, Montréal, les Herbes rouges, coll. «Lecture en vélocipède», 1982, $175 \mathrm{p}$.

Intérieurs, Montréal, les Herbes rouges, 1984, 38 p.

Transit, Montreal, les Herbes rouges, 1984, 45 p.

Entre raison et déraison. Essais, Montréal, les Herbes rouges, 1987, 164 p.

\section{I.2 - Mémoire et thèse}

«Discours du moi et discours de l'autre dans les Oranges sont vertes de Claude Gauvreau», mémoire de maitrise, Montréal, Université de Montréal, 1976, $111 \mathrm{p}$.

«Essai d'une écriture plurielle - Pour une mise en situation de textes concomitants», thèse de doctorat, Sherbrooke, Université de Sherbrooke, 1981, $523 \mathrm{p}$.

\section{I.3 - Ouvrages en collaboration}

\section{I.3.1. - Livres}

«Préface» à la Nef des sorcières, Montréal, Quinze, 1976, p. 7-13 [en collaboration avec Nicole Brossard].

«L'échantillon», la Nef des sorcières, Montréal, Quinze, 1976, p. 31-38.

«Claude Gauvreau, poète et mythocrate, Jean Marchand, VLB éditeur», Livres et auteurs québécois, 1979, p. 235-237.

«La vieille petite fille», Célibataire pourquoi pas?, Serge Fleury éditeur, 1981, p. $133-140$. 
«La lenteur, le cri, l'autonomie», Marguerite Duras à Montréal, textes réunis et présentés par S. Lamy et A. Roy, Montréal, Éditions Spirale, 1981, p. 91-94.

«Écarts: pour une approche des différences», l'Oiseau-chat. Roman-enquête sur l'identité québécoise, Montréal, la Presse, 1983, p. 213-221.

«Écrire - l'amour, dit-elle - Ah! Harlequin, dit-il», Écrire l'amour, Montréal, l'Hexagone, 1984, p. 44-47 [XIe Rencontre québécoise internationale des écrivains].

«La Charge de l'orignal épormyable, fiction dramatique de Claude Gauvreau», Dictionnaire des auvres littéraires du Québec, tome IV, 1960-1969, Montréal, Fides, 1984, p. 149-150.

«L'Étalon fait de l'équitation, drame de Claude Gauvreau», Dictionnaire des auvres littéraires du Québec, tome IV, 1960-1969, Montréal, Fides, 1984, p. 320.

«Une femme médecin raconte..., roman de Béatrix [pseudonyme de Gabrielle Paquin née Grandbois]», Dictionnaire des auvres littéraires du Québec, tome IV, 1960-1969, Montréal, Fides, 1984, p. 905-906.

«L'écriture est lenteur», Essais québécois 1837-1983, Montréal, Hurtubise HMH, 1984, p. 607-608 [paru d'abord dans Dérives, no $35,4^{e}$ trimestre 1982, p. 58-60].

«Situation actuelle de la poésie», Choisir la poésie, Trois-Rivières, Écrits des Forges, 1986, p. 37-39.

«L'autre femme», Installations/Fictions, Montréal, NBJ, 1986, [n.p.]. Dessins de Betty Goodwin.

«Le déplacement du symbolique», texte paru en traduction dans Amazing Space, collectif d'auteurs, edited by Shirley Neuman and Smaro Kamboureli, Longspoon/Newest, Alberta, 1986.

«Fiction et métissage ou écrire l'imaginaire du réel», l'Écriture: lieu théorique et pratique du changement, Actes du colloque de Charleroi, Nouvelle Barre du jour, nos 201-202, novembre 1986, p. 63-78.

«Éloge de la mémoire des femmes», la Théorie un dimanche, Montréal, Éditions du Remue-ménage, 1988, p. 175-191 [en collaboration avec Nicole Brossard, Louky Bersianik, Louise Cotnoir, Louise Dupré, Gail Scott].

«Ceci n'est pas un lac [extrait]», ibid., p. 193-201.

«Sur fond d'ennui», Enfances et jeunesses, Montréal, les Entreprises Radio-Canada, 1988 , p. $219-227$.

«Un point névralgique: l'irrationalité», Folie, Mystique, Poésie (textes réunis par Raymond Hervieux), Québec, Éd. du GIFRIC, coll. «Nœud», 1988, p. 43-50.

\section{I.3.2 - Articles}

«L'avant-garde», le Nouveau Cahier, 2 mars 1967, p. 8 [en collaboration avec Marcel Saint-Pierre].

«À propos de... Roland Giguère», la Barre du jour, nos 11-12-13, décembre 1967. mai 1968, p. 163-169 [propos recueillis par France Théoret et Jean Stafford].

«Le matriarcat québécois analysé par les reines du foyer», les Têtes de pioche, no 1 , mars 1976, p. 1, 3, 8 [en collaboration avec Michèle Jean]. 
«Révélatrices: femmes et photos», la Nouvelle Barre du jour, nos 136-137, mars 1984, p. 1-172 [en collaboration avec Germaine Beaulieu, Micheline de Jordy, Denise Marchand].

I.4 - Articles (essais critiques, proses et poèmes) de périodiques et de journaux

«Contes pour lecteurs attardés», le Cahier, 15 septembre 1966, p. 4.

«Breton ravagea tout: y compris les conséquences», le Nouveau Cahier, 13 octobre 1966, p. 1.

«Hypothèses. Dans une démarche littéraire, le chemin parcouru compte beaucoup plus que le point d'arrivée», le Cahier, 27 octobre 1966, p. 1-3.

«Jet 1», la Barre du jour, no 9, janvier-février 1967, p. 22-23.

«Jet 2», la Barre du jour, no 9, janvier-février 1967, p. 24-25.

«La Barre du jour. Exploitation de l'imagination québécoise», le Nouveau cahier, 16 février 1967, p. 5.

«Les paradoxes de la culture», le Nouveau Cahier, 2 mars 1967, p. 1.

«Texte pour la voix haute», la Barre du jour, no 14, juin-juillet 1968, p. 3-5.

«Présentation de Lettre à un fantôme de Claude Gauvreau», la Barre du jour, nos 17 18-19-20, janvier-août 1969 , p. 342-343.

«Présentation de trois objets dramatiques de Claude Gauvreau», la Barre du jour, nos 17-18-19-20, janvier-août 1969, p. 375 .

«Notes sur la figure: En attendant Godot», Critère, nos 6-7, septembre 1972, p. 295-301 [signé France Saint-Pierre].

«Le triomphe des forces concentrationnaires. Note sur l'Hiver de force de Réjean Ducharme», Stratégie, no 9, été 1974, p. 81-84.

«Au retour du refoulé, la fiction», Chroniques, nos 6-7, juin-juillet 1975, p. 109 112.

«Dépendances», la Barre du jour, no 50, hiver 1975, p. 28-29.

«The Sample», Exile, vol. 14, no 1, 1976, p. 107-112 [traduction de David Ellis]. «Comment le privé est politique», Chroniques, no 13 , janvier 1976, p. 30-36.

«Je baise à tout vent», les Têtes de pioche, no 2, avril 1976, p. 7.

«Pour la cause des femmes?» les Têtes de pioche, no 3, mai 1976, p. 4.

«La petite fille sage», les Têtes de pioche, no 4, juin 1976, p. 3.

«La femme et l'écriture», Liberté, nos 106-107, juillet-août 1976, p. 122-125.

«Masochisme féminin et fascisme», les Têtes de pioche, no 5, septembre 1976, p. 4.

«Cochonnerie», la Barre du jour, nos 56-57, mai-août 1977, p. 12-19.

«Fragment d'une lettre sur la fiction à mes amies étrangères», la Barre du jour, nos 56-57, mai-août 1977 , p. 196-200.

«Présentation des cuvres créatrices de Gauvreau», la Nouvelle Barre du jour, no 58, septembre 1977, p. 95-99.

«Virginia Woolf. Journal d'un écrivain», la Nouvelle Barre du jour, no 59, octobre 
1977, p. 82-84.

«Garbage», A Room of one's own, vol. IV, nos 1-2, 1978, p. 55-59 [traduction de Josée M. Leblond].

«Scénographie», Sorcières, no 14, 1978, p. 10-12.

«Pour une lecture critique des textes de femmes», la Nouvelle Barre du jour, no 66, mai 1978, p. 76-79.

«Résister au codage», le Devoir, 3 juin 1978, p. 36.

«Métal jaune», la Nouvelle Barre du jour, nos 68-69, septembre 1978, p. 175-178.

«Traduction», Interprétation, nos 22-23, automne 1978-printemps 1979, p. 251254.

«Feu de langue», les Stratégies du réel/The story so far 6, Montréal, la Nouvelle Barre du jour et Toronto, Coach House Press, 1979, p. 198-217 [accompagné d'une traduction de Patricia Claxton].

«Cartes postales d'Acapulco!», la Nouvelle Barre du jour, no 77, avril 1979, p. 43-47.

«Le style et le désirn, Spirale, no 1, septembre 1979, p. 7 [sur le Centre blanc de Nicole Brossard].

«La place du corps», Spirale, no 1, septembre 1979, p. 12 [sur la Peur surtout au Théâtre expérimental des femmes].

«Un cas exemplaire», Spirale, no 1, septembre 1979, p. 14 [sur Seul, comme Kafka de Marthe Robert].

«La guerrière», Possibles, automne 1979, p. 101-106.

«Qu'est-ce qu'écouter? Entretien avec Patrick Straram le Bison ravi», Spirale, no 2, octobre 1979, p. 3, 15.

«Les fragments de l'histoire», Spirale, no 2, octobre 1979, p. 10 [sur Pedro Paramo de Juan Ruffo].

«Une voix de femme à propos de viol», Spirale, no 2, octobre 1979, p. 16 [sur Mourir à tue-tête d'Anne-Claire Poirier].

«Un certain féminisme français», Spirale, no 3, novembre 1979, p. 11 [sur Ni tout à fait la même ni tout d fait une autre... de Flora Groult].

«Qu'est-ce qu'écouter? [2] Entretien avec Patrick Straram le Bison ravi», Spirale, no 3, novembre 1979, p. 13.

«Une littérature de bon ton», Spirale, no 4, décembre 1979, p. 5 [sur Mélano de Marie Lafleur et les Cloisons de Solange Lévesque].

«Générations», Dérives, no 26, 1980, p. 39-44 [extraits].

«Une histoire de mère et de séparation», Spirale, no 5 , janvier 1980, p. 3 [sur l'Hippocanthrope de France Vézina].

«Un livre rare», Spirale, no 5, janvier 1980, p. 8 [sur l'Atelier de Claire Lejeune]. «Pour une télévision poétique», Spirale, no 6, février 1980, p. 6. 
«Entre l'excès du verbe et de la communication», Spirale, no 6, février 1980, p. 7 [sur le Pique-nique sur l'Acropole de Louky Bersianik].

«Des fragments levés à même le tissu quotidien», la Nouvelle Barre du jour, no 88, mars 1980, p. 97-107.

"L'autre langage télévisuel. Interview avec Louise Guay à propos de l'émission Les Térieurs», Spirale, no 8, avril 1980, p. 14-15.

«Kaléidoscope», le Devoir, 19 avril 1980, p. 26.

«Qu'est-ce que le sens?» Spirale, no 9, mai 1980, p. 12 [sur les Tyrannies de l'intimité de Richard Sennett].

«L'implicite et l'explicite de la nouvelle écriture», la Nouvelle Barre du jour, nos $90-91$, mai 1980 , p. $165-170$.

«Nœud», la Nouvelle Barre du jour, nos 90-91, mai 1980, p. 171-172 [extrait].

«La marche», la Nouvelle Barre du jour, nos 92-93, juin 1980, p. 223-226.

«Un livre impatient», Spirale, no 10, juin 1980, p. 1, 6 [sur la Mère des herbes de Jovette Marchessault].

«A propos de la critique féministe», Spirale, no 11, septembre 1980, p. 2 [réponse à une lettre de Gloria Feman Orenstein].

«La transparence», Spirale, no 11, septembre 1980 , p. 10.

«Faut-il mourir?», Spirale, no 11, septembre 1980, p. 12 [sur Mars de Fritz Zorn].

«La loi comme noud dans les Oranges sont vertes de Claude Gauvreau», la Nouvelle Barre du jour, no 94, septembre 1980, p. 57-79.

«Le désir de vagabondage», Spirale, no 12 , octobre 1980, p. 1,4.

«Grey painting. Peinture grise», Canadian Forum, no 60, octobre 1980, p. 22-23 [traduction de Patricia Smart].

«Les mots circulent», Spirale no 14, décembre 1980, p. 15 [ sur Culture et littérature: perspectives. Colloque Québec-Belgique].

«A celles qui ont trop travaillé», Anthologie 80, le Castor astral/l'Atelier de l'agneau, 1981, p. 283-284.

«La vieille petite fille», Interprétation, no 24, 1981, p. 117-122.

«Le corps s'expérimente», Spirale, no 15, janvier 1981, p. 15 [sur Landes de Chantal Chawaf].

«Le réalisme de l'inconscient», Spirale, no 16, février 1981, p. 3 [sur Kati of course de Julien Bigras].

«Ne pas peser sur la terre», la Nouvelle Barre du jour, nos 100-101, mars 1981, p. 126-128.

«Nœud», la Nouvelle Barre du jour, no 102, avril 1981, p. 23-26 [extrait].

«Penser le tiers exclu», Spirale, no 18, avril 1981, p. 4 [sur l'Issue de Claire Lejeune].

«Mon héroìne», Spirale, no 18, avril 1981, p. 4. [sur les Lundis de l'histoire des femmes au Théâtre expérimental des femmes]. 
«Les rouages de la pensée», Spirale, no 18, avril 1981, p. 5 [sur l'Eté 80 de Marguerite Duras].

«Du côté des mutantes», Spirale, no 19, mai 1981, p. 5 [sur De l'amour lesbien de Geneviève Pastre].

«Répercuter les premiers mots. Interview avec Jovette Marchessault», Spirale, no 20 , juin 1981, p. 18.

«La crise et le désir», Spirale, no 21, septembre 1981, p. 7 [sur Chroniques souterraines de Lise Harou].

«Quand l'ignorance fait souffrir», Spirale, no 21, septembre 1981, p. 7 [sur la Traversée des apparences de Virginia Woolf].

«Un livre tautologique», Spirale, no 21, septembre 1981, p. 21 [sur la Nourriturenévrose de Michèle Declerck et Jeanne Boudouard].

«Gifler la mère», Spirale, no 21, septembre 1981, p. 23 [sur Éloge du cosmopolitisme de Guy Scarpetta].

«Petite Galerie», la Nouvelle Barre du jour, no 106, octobre 1981, p. 62-67.

«Écrans: entre folie et raison», le Devoir, 28 novembre 1981, p. 29.

«Quand la mémoire dérive trop», Estuaire, no 18, hiver 1981, p. 121-130.

«Habitat II», Rampike, vol. 2, nos 1-2, 1982, p. 11.

«Corps et violence», Spirale, no 22, février 1982, p. 4 [sur Babelle 1. Après le déluge de Renaud Longchamps].

«Introduction à un journal», Spirale, no 23, mars 1982, p. 5 [sur Journal de Virginia Woolf].

«Les bonnes intentions», Spirale, no 24, avril 1982, p. 4 [sur Un moulin, un village, un pays de Gilles Raymond].

«Un feu roulant», Spirale, no 24, avril 1982, p. 15 [sur «... Quand j'y ai dit ça... à parti à rirex de Léo Lévesque].

«L'aliénation par le bienfait», Spirale, no 25, mai 1982, p. 7 [sur Avant-garde suivi de Souvenirs sur Brecht de Marieluise Fleisser].

«Avez-vous lu Jeanne Hyvrard?», Spirale, no 26, juin 1982, p. 7 [sur le Corps défunt de la comédie de Jeanne Hyvrard].

«La mère peut-elle être moderne?», la Nouvelle Barre du jour, no 116, septembre 1982, p. 47-50.

«Elle n'est pas noire, ni antillaise. Entretien avec Jeanne Hyvrard», Spirale, no 27, septembre 1982, p. 15.

«Mexico magique», Mensuel 25, nos 64-65-66, septembre-octobre-novembre 1982, [n.p.].

«Prendre la parole quand on est femme», Québec français, no 47, octobre 1982, p. 36-37.

«Somewhere over the rainbow», Spirale, no 28, octobre 1982, p. 3 [sur l'Amour langue morte de Solange Lévesque].

«Joyce, écrivain», Spirale, no 28, octobre 1982, p. 10 [sur James Joyce de Richard Ellmann]. 
«Le sens de la formule», la Nouvelle Barre du jour, nos 118-119, novembre 1982, p. 77-82 [Sur Nicole Brossard].

«Le goût de tuer?», Spirale, no 29, novembre 1982, p. 6 [sur l'Armoire de Pantagruel de Claude Jasmin].

«La passion du vide», Spirale, no 29, novembre 1982, p. 6 [sur Près du caur sauvage de Clarice Lispector].

«Qui lira jugera», Spirale, no 30, décembre 1982, p. 15 [sur Erreur sur la personne de Serge Provencher].

«Habitat II», Rampike, vol. 3, no 2, 1983, p. 17.

«Habitat III», la Nouvelle Barre du jour, nos 122-123, février 1983, p. 192-193.

«Un roman sans ombres», Spirale, no 31, février 1983, p. 3 [sur Picture Theory de Nicole Brossard].

«En bref», Spirale, no 32, mars 1983, p. 8 [sur Une histoire gitane de Hélène Rioux].

«En bref», Spirale, no 32, mars 1983, p. 14 [sur Sortie d'usine de François Bon].

«L'autobiographie exemplaire?», Spirale, no 32, mars 1983, p. 15 [sur Ma vie comme rivière, tome 2 , de Simone Monet Chartrand].

«Histoire de petits pouvoirs», Spirale, no 33, avril 1983, p. 13 [sur Amours profanes de Joyce Carol Oates].

«Ce grand vide qu'on dit intérieur», Etudes littéraires, vol. XVI, no 6, avril 1983, p. 163-166.

«En bref», Spirale, no 34, mai 1983, p. 8 [sur Journal de Mathieu et Lettres à Simone Jolivet de Jean-Paul Sartre, revue les Temps modernes no 434, le Sujet de la science est-il sexué? de Luce Irigaray, revue les Temps modernes, no 436].

«De la chair à la langue», la Vie en rose, no 11, mai 1983, p. 53-56.

«Une fable bien faite», Spirale, no 34, mai 1983, p. 9 [sur la Ville aux gueux de Pauline Harvey].

«En bref», Spirale, no 35, juin 1983, p. 4 [sur Etat des lieux en collaboration].

«En bref», Spirale, no 35, juin 1983, p. 6 [sur le Long des paupières brunes de Rolande Ross].

«Une longue plainte», Spirale, no 35, juin 1983, p. 18 [sur $D u$ lieu des voyages de France Ducasse].

«Fin d'après-midi», la Vie en rose, no 12, juillet 1983, p. 47.

«L'analyse systématique en procès», Spirale, no 36 , septembre 1983, p. 5 [sur Un matriarcat en procès de Janine Boynard-Frot].

«La continuité/la discontinuité», Spirale, no 36, septembre 1983, p. 11 [sur Blues clair: Tea for one/ no more tea de Patrick Straram le Bison ravi].

«En bref», Spirale, no 37, octobre 1983, p. 6 [sur l'Écrivain devant son auvre de Donald Smith].

«Dire l'époque», Spirale, no 37, octobre 1983, p. 11 [sur Je suis ce que je suis de François Charron]. 
«En bref», Spirale, no 37, octobre 1983, p. 12 [sur le Poids du réel, la souffrance de Denis Vasse].

«Les Québécois en France», Spirale, no 38, novembre 1983, p. 3 [sur Laura Laur de Suzanne Jacob].

«En bref», Spirale, no 39, décembre 1983, p. 2 [sur $\grave{A}$ voix basse de Gilles Archambault].

«Traces d'un travail inégal», Spirale, no 39, décembre 1983, p. 5 [sur Revoir le rouge d'Hugues Corriveau].

«En bref», Spirale, no 39, décembre 1983, p. 9 [sur Dérives, nos 37-38-39, Nouvelles brésiliennes].

«Le vide antérieur», Estuaire, nos 32-33, 1984, p. 130-131.

«En bref», Spirale, no 40, février 1984, p. 16 [sur Un printemps froid de Danièle Sallenave].

«Grandeurs et misères du roman», Spirale, no 41, mars 1984, p. 3 [sur Maryse de Francine Noël,].

«Habitat V», la Nouvelle Barre du jour, nos 136-137, mars 1984, p. 122-124.

«Lire Huguette Gaulin», Spirale, no 42, avril 1984, p. 9 [sur Lecture en vélocipède d'Huguette Gaulin].

«En bref», Spirale, no 42, avril 1984, p. 9 [sur la Malentendue de Nicole Houde].

«Une méditation amoureuse», Spirale, no 43, mai 1984, p. 3 [sur l'Existence de Carole Massé].

«Larmes», Passages, no 3, printemps-été 1984, p. 57.

«Le grand écart», la Nouvelle Barre du jour, no 140, juin 1984, p. 114.

«Cherchez la femme!», Spirale, no 44, juin 1984, p. 8 [sur Histoire de l'impossible pays de François Hébert].

«En bref», Spirale, no 44, juin 1984, p. 8 [sur Black Diva de Jean-Paul Daoust].

«La transformation du roman québécois entre 1965 et 1980», Nova Renascença, vol. IV, julho-setembro 1984, p. 292-299 [paru aussi dans Francofonia studi $e$ ricerche sulle letterature di langua francese, no 7, autunno 1984, p. 83-93].

«L'avenir à l'infinitif», la Nouvelle Barre du jour, no 141, septembre 1984, p. 5556.

«Peut-on démoder la modernité?», Spirale, no 45, septembre 1984, p. 4 [sur la Tyrannie de l'imprimé de Marthe Robert].

«L'indifférence généralisée», Spirale, no 46, octobre 1984, p. 10 [sur l'Ère du vide de Gilles Lipovetsky].

«En bref», Spirale, no 46, octobre 1984, p. 12 [sur l'Homme éclaté de PierreYves Pépin].

«La métaphore de la demeure», Spirale, no 48, décembre 1984, p. 8 [sur Éthique de la différence sexuelle de Luce Irigaray].

«En bref», Spirale, no 51, avril 1985, p. 13 [sur Auditions musicales certains soirs d'été de Jeanne Hyvrard]. 
«Croyez-vous au destin?», Spirale, no 52, mai 1985 p. 8 [sur Hommes et Femmes d'Annie Leclerc].

«La parole mitoyenne», Spirale, no 53, juin 1985, p. 11 [sur l'Eil de la lettre de Claire Lejeune].

«L'autre femme», l'Arbre à paroles, no 55, septembre 1985, p. 128-129.

«Prendre le risque d'une nouvelle globalité/souveraineté du sujet parlant», la Nouvelle Barre du jour, no 157, septembre 1985, p. 7-11.

«La posture de l'écrivain», Mabius, no 26, automne 1985, p. 103-106 [sur Littérature l' imposture d'André Beaudet].

«Sans visage», Possibles, vol. 10, no 1, automne 1985, p. 157-164.

«Écriture au féminin et institutions», Dialogues et culture, no 27, 1985, p. 223225.

«Des nerfs à vif ou vivisection de langue», Estuaire, no 38,1986, p. 83-102.

«La turbulence intérieure», la Nouvelle Barre du jour, no 172, mars 1986, p. 73 79.

«L'homme qui peignait Staline (work in progress)», la Nouvelle Barre du jour, no 181 , septembre 1986 , p. $83-95$; no 184 , octobre 1986 , p. 49-61; no 187 , novembre 1986 , p. $81-92$, no 193 , février 1987 , p. 67-79.

«La voix poétique», Estuaire, nos 40-41, 1986, p. 114-117.

«La marche», Vagabondages, no 66, janvier-février-mars 1987, p. 34-35 [paru aussi dans Jean Royer, la Poésie québécoise contemporaine. Anthologie, Montréal/ Paris, l'Hexagone, 1987, p. 193-195].

«Résolument le féminin», Spirale, no 71, été 1987, p. 10 [sur d'elles et Quand je lis je $m$ 'invente de Suzanne Lamy].

«Onze ans», Brèves, nos 24-25, printemps 1987, p. 75-85.

«La fascination», Possibles, vol. 12, no 1, hiver 1988, p. 27-35.

«Visages», Trois, vol. III, no 3, printemps-été 1988, p. 165.

«Joumal intime», la Parole métèque, no 6, été 1988, p. 19.

«Ce qui est», Estuaire, no 50, automne 1988, p. 95-96.

I.5 - Communications, entretiens radiophoniques

«Le je tremblant des femmes publiées aux Éditions de l'Hexagone», texte lu à Toronto, au colloque 25 e anniversaire des Éditions de l'Hexagone, en 1979 [inédit].

«Il n'y eut jamais assez de mots», texte lu à Montréal, à la Société de philosophie de l'université de Montréal, en 1980 [inédit].

«Les nouvelles formes de la subjectivité», texte lu à Montréal, au colloque les Herbes rouges, 1987 [inédit].

Entrevue avec Gilles Archambault, le Jardin secret, Radio-Canada MF, 30 décembre 1987.

Entrevue avec Claude Lévesque: à propos de Entre raison et déraison, les Idées à l'essai, Radio-Canada MF, 19 avril 1988. 
«La fiction de l'ange» dans Miss Morphose, spectacle en collaboration pour la Foire du livre féministe de Montréal, 16-17-18 juin 1988.

II - Etudes sur l'auteure et son œuvre

GRUSLIN, Adrien, «La Nef des sorcières, un spectacle nécessairement féministe au TNM», le Devoir, 28 février 1976, p. 17.

VILLEMAIRE, Yolande, "Autour de la Nef des sorcières», Jeu, no 2, printemps 1976 , p. 16-21.

DIONNE, André, «La Nef des sorcières», Lettres québécoises, no 3, septembre 1976, p. 15.

SAINT-JACQUES, Denis, «La Nef des sorcières ou les paramécides massacrées», Lettres québécoises, no 3, septembre 1976, p. 17-19.

BONENFANT, Joseph, «Bloody Mary», Livres et auteurs québécois, 1977, p. 157 160.

CHOUINARD, Ivan, «Un projet de roman sur son enfance», l'Avenir, no 15 , avril 1978 , p. A2.

MELANÇON, Robert, «Un livre de vérité», le Devoir, 15 avril 1978, p. 35.

ROY, Monique, «Une voix pour Odile. "J'écris au singulier"», le Devoir, 15 avril 1978 , p. 35.

GAGNON, Madeleine, «Une voix pour Odile», Voix \& images, vol. IV, no 1, septembre 1978, p. 143-146.

ROY, Monique, «Une voix pour Odile», les Cahiers de la femme, automne 1978, p. 110-111.

GIROUX, Robert, "Une voix pour Odile», Livres et auteurs québécois, 1978, p. 147-150.

HAECK, Philippe, Naissances. De l'écriture québécoise, Montréal, VLB éditeur, 1979, p. 221-230.

ROYER, Jean, «Célébrations au TNM. Le théâtre musical de la fête», le Devoir, 7 mars 1979, p. 16.

MELANÇON, Robert, «Des Trobairitz à France Théoret», le Devoir, 14 avril 1979, p. 21.

DUPRE, Louise, «L'urgence d'une critique féministe», le Devoir, 28 avril 1979, p. 26.

GIGUĖRE, Richard, «Romans», University of Toronto Quarterly, vol. XLVIII, no 4, Summer 1979, p. 359.

CORRIVEAU, Hugues, «Poésie: des lèvres et des vertiges», la Nouvelle Barre du jour, no 81, septembre 1979, p. 87-90.

HAECK, Philippe, «La souffrance infinie», Spirale, no 1, septembre 1979, p. 11.

GIGUĖRE, Richard, «Vertiges», Livres et auteurs québécois, 1979, p. 105-106.

STRARAM LE BISON RAVI, Patrick, «Essentiellement écrivante... France Théoret», le Livre d'ici, vol. V, no 52, $1^{\text {er }}$ octobre 1980, [n.p.].

OUELLETTE-MICHALSKA, Madeleine, «Nécessairement putain», le Devoir, 15 novembre 1980 , p. 23. 
GAULIN, André, «Nécessairement putain», Québec français, no 40, décembre 1980, p. 15.

NEPVEU, Pierre, «Nicole Brossard et France Théoret: la pensée/l'impensable», Lettres québécoises, no 20, hiver 1980-1981, p. 24-27.

HAECK, Philippe, «Nécessairement putain», Livres et auteurs québécois, 1980, p. 98-100.

LAMARRE, André, «Nécessairement putain», Livres et auteurs québécois, 1980, p. $135-137$.

BEAULIEU, Michel, «Vertiges», Apostrophe magazine, nos 6-7, été 1981.

OUELLETTE-MICHALSKA, Madeleine, «Vivre comme on Ecrit?», le Devoir, 15 mai 1982, p. 19.

ROYER, Jean, «France Théoret. Les sciences exactes de l'être», le Devoir, 15 mai 1982, p. 19.

MARTEL, Réginald, «Un roman de France Théoret. Trancher le nœud des générations», la Presse, 29 mai 1982, p. C3.

BEAULIEU, Michel, «France Théoret, des mémoires fascinantes», le Livre d'ici, vol VII, no 47, 25 août 1982, [n.p.].

MASSE, Carole, «Nous parlerons comme on écrit», les Cahiers de la femme, vol. IV, no 2 , hiver 1982 , p. 97-98.

HAYWARD, Annette, «Nous parlerons comme on écrit», Québec français, no 48, décembre 1982, p. 3.

SALESSE, Michèle, «Nous parlerons comme on écrit», Lettres québécoises, no 28 , hiver $1982-1983$, p. 23-24.

DORION, Hélène, «Nous parlerons comme on écrit», Livres et auteurs québécois, 1982 , p. $79-80$.

LAMY, Suzanne, «Des résonnances de la petite phrase: "Je suis un nœud" de France Théoret», Féminité, Subversion, Ecriture, Montréal, Editions du Remueménage, 1983, p. 139-149.

DESJARDINS, Normand, «Nous parlerons comme on écrit», Nos livres, vol XIV, janvier 1983, p. 54-55.

BEAUSOLEIL, Claude, «France Théoret, nécessairement les mots», les Livres parlent, Trois-Rivières, Écrits des Forges, 1984, p. 207-208.

FOURNIER, Danielle, «Entretien avec France Théoret», Mabius, no 23, 1984, p. 71-77.

JONASSAINT, Jean, «Écrire un thêâtre musical. Transit. Entretien avec Micheline Coulombe Saint-Marcoux et France Théoret», Dérives, nos 44-45, 1984, p. 74-84.

ROYER, Jean, «Une voix brûlante», le Devoir, 8 septembre 1984, p. 13.

ROYER, Jean, «La $15^{\mathrm{e}}$ saison de l'Eskabel débute avec Transit», le Devoir, 20 septembre 1984 , p. 9.

ROYER, Jean, «Transit. La modernité mise en musique», le Devoir, 29 septembre 1984, p. 32. 
GINGRAS, Claude, «Huit heures d'attente, toute une vie. Transit, un thêatre musical», la Presse, 29 septembre 1984, p. E8.

GINGRAS, Claude, «Transit. Du visuel avec un peu de musique», le Devoir, 4 octobre 1984, p. 26.

ROYER, Jean, «Transit: du théâtre musical superbe à l'Eskabel», le Devoir, 6 octobre 1984, p. 30.

FERLAND, Isabelle, «A la recherche du corps perdu», Continuum, 9 octobre 1984, p. 18.

ANDRĖS, Bernard, «Musique thêâtralisée?», Spirale, no 48, décembre 1984, p. 12.

«Entretien avec France Théret», Foldaan (anthologie), no 5, 4e trimestre 1984, p. 63-66 [numéro sur «Sept poètes québécois»].

ALONZO, Anne-Marie, «France Théoret trois fois», la Vie en rose, no 22, décembre 1984-janvier 1985, p. 61.

ROBERT, Dominique, «La création ou le degré de l'absolu. Entretien avec France Théoret et Micheline Coulombe Saint-Marcoux», Sortie, décembre 1984janvier 1985, p. 23.

BONENFANT, Joseph et André GERVAIS, «Le fantasme de la $B J$, c'est la théorie. Entrevue avec France Théoret», Voix \& images, vol. X, no 2, hiver 1985, p. 87-92.

FOURNIER, Danielle, «Intérieurs et Transit», Arcade, no 9, février 1985, p. 8586.

TRUDEL, Serge, «Nécessairement putain», Nos livres, vol. XVI, nos 6038-6095, février 1985, p. 41.

D'ALFONSO, Antonio, «Transit», Nos livres, vol. XVI, nos 6096-6139, mars 1985, p. 38-39.

GIGUĖRE, Richard, «Les Herbes rouges, encore!», Lettres québécoises, no 37 , printemps 1985 , p. 47-49.

MUIR, Michel, «Imposture poétique: France Théoret», Poètes ou Imposteurs, Montréal, Louise Courteau, éditrice, 1985, p. 51-61.

DRAPEAU, Renée-Berthe, «Féminins singuliers. Pratiques d'écriture: Brossard, Théoret», mémoire de maîtrise, Sherbrooke, Université de Sherbrooke, 1985, 142 p.

SMART, Patricia, «Quand la fille du bar se met à parler: la poésie de France Théoret», Dalhousie French Studies (numéro spécial sur la poésie québécoise depuis 1975), 1985, p. 153-162.

TUAILLON, Gisèle, «France Théoret ou la parole empêchée», thèse de DEA (sous la direction de R. Barny), Université de Franche-Comté, Faculté des lettres et sciences humaines de Besançon, 1985, $164 \mathrm{p}$.

BROCHU, André, «Des fous et des autres», Voix \& images, vol X, no 3, printemps 1985, p. 179-187.

TEMPLE, Frédéric-Jacques, «Le vide intérieur», Québec vivant. Anthologie, Paris, Sud, coll. «Domaine étranger», 1986, p. 205-207. 
BONENFANT, Joseph, «Ce qui suffit à l'Cil», Urgences, no 15, octobre 1986 , p. 91.

CHASSAY, Jean-François, «France Théoret humoriste?», Estuaire, no 38, 1986, p. 49-54.

CORRIVEAU, Hugues, «Des yeux qui écrivent», Estuaire, no 38, 1986, p. 13-22.

DUPRE, Louise, «Qui parle?»; Estuaire, no 38, 1986, p. 41-48.

GAUDET, Gérald, «Les fictions du réel», Estuaire, no 38, 1986, p. 11-12.

GAUDET, Gérald, «Une écriture responsable (entretien avec France Théoret)», Estuaire, no 38, 1986, p. 103-118.

LEJEUNE, Claire, «Clefs pour une correspondance à quatre voix», Estuaire, no 38, 1986, p. 31-40.

NEPVEU, Pierre, «Intérieurs d'une pensée», Estuaire, no 38, 1986, p. 23-30.

RACINE, Robert, «France Théret. Bibliographie», Estuaire, no 38, 1986, p. 121131.

SMART, Patricia, «Un réalisme moderne: l'approche du réel», Estuaire, no 38, 1986 , p. $55-72$.

SIMON, Sherry, «Éloge de la difficulté», Spirale, no 78, avril 1988, p. 7.

PRÉVOST, Francine, «Ex libris [...] Entre raison et déraison», Trois, vol. III, no 3, printemps-été 1988 , p. 176.

BOIVIN, Jean-Roch, «Ceci n'est pas une critique et la théorie a ses limites», le Devoir, 25 juin 1988, p. D3.

GAUDET, Gérald, «Les tremblements de la raison», le Devoir, 25 juin 1988, p. D3.

GIGUĖRE, Richard, André MARQUIS et Joseph BONENFANT, «Les Herbes rouges, 1968-1988: persister. et se maintenir» (interview avec Normand de Bellefeuille, Carole Massé, André Roy et France Théoret), Lettres québécoises, no 51 , automne 1988 , p. $10-17$.

THÉRY, Chantal, «C'est dans la fiction au féminin que se joue le devenir du féminisme comme philosophie», Lettres québécoises, no 51, automne 1988, p. 49-50. 